\title{
Acyl-CoA:diacylglycerol acyltransferase 1 inhibition in the small intestine increases plasma transaminase activity via the activation of protein kinase $C$ pathway
}

\author{
Hideaki Yokoyama1,2, Taku Masuyama1, Yuki Tanaka1, lori Tsubakihara1, \\ Kazuma Kondo ${ }^{1}$ and Kouichi Yoshinari ${ }^{2}$ \\ ${ }^{1}$ Toxicology Research Lab., Central Pharmaceutical Research Institute, JAPAN TOBACCO INC., \\ 1-13-2 Fukuura, Kanazawa-ku, Yokohama-city, Kanagawa 236-0004, Japan \\ ${ }^{2}$ Department of Molecular Toxicology, School of Pharmaceutical Sciences, University of Shizuoka, \\ 52-1 Yada, Suruga-ku, Shizuoka-city, Shizuoka 422-8526, Japan
}

(Received September 2, 2021; Accepted October 22, 2021)

\begin{abstract}
Acyl-CoAdiacylglycerol acyltransferase 1 (DGAT1) is a key enzyme in the fat absorption step in enterocytes. We previously reported that the pharmacological inhibition of DGAT1 increased plasma alanine aminotransferase (ALT) and aspartate aminotransferase (AST) activity in corn oil-loaded rats without any sign of hepatotoxicity. In this study, we investigated this mechanism. We found that this elevation occurred only during the pharmacologically active period of a DGAT1 inhibitor and the magnitude did not depend on the volume of corn oil. In addition, this elevation was not accompanied by increases in ALT or AST mRNA levels in the small intestine and liver. To clarify a lipid component responsible for this elevation, rats were treated with free fatty acids instead of corn oil and no plasma ALT elevation was observed. Next, rats were pretreated with inhibitors of monoacylglycerol acyltransferase 2 and intestinal microsomal triglyceride transfer protein instead of the DGAT1 inhibitor, but no plasma ALT elevation was observed after corn oil loading. Since the results suggested a possible role of diacylglycerol (DAG), which activates protein kinase C (PKC), we measured PKC activity in the small intestine and found that the activity was increased by treatment with the DGAT1 inhibitor and corn oil. Moreover, rats pretreated with a PKC inhibitor in combination with the DGAT1 inhibitor showed suppression of plasma ALT elevation. Taken together, the present results suggest that DAG accumulation induced by pharmacological DGAT1 inhibition and resultant PKC activation in enterocytes are involved in the increase in plasma ALT and AST activity in rats.
\end{abstract}

Key words: Alanine aminotransferase, Fatty acids, PKC inhibitor, Hepatotoxicity, Markers of liver injury, Fat absorption

\section{INTRODUCTION}

Acyl-CoA:diacylglycerol acyltransferase (DGAT) is a major enzyme that synthesizes triacylglycerol (TAG) from fatty acyl-CoAs and diacylglycerol (DAG). DGAT isozymes, DGAT1 and DGAT2 have been identified. DGAT1 is highly expressed in the small intestine while DGAT2 is highly expressed in the liver and fat tissues (Cases et al., 1998, 2001). Recently, Tomimoto et al. (2015a, 2015b) discovered a new selective DGAT1 inhibitor, JTT-553, [trans-5'-(4-amino-7,7-dimethyl-2-trifluo-
romethyl-7H-pyrimido[4,5-b][1,4] oxazin-6-yl)-2',3'dihydrospiro(cyclohexane-1,1-inden)-4-yl]acetic acid monobenzenesulfonate, with human DGAT1 inhibitory activity. This compound suppressed postprandial fat absorption in the small intestine and fat synthesis in the adipose tissue, and thus reduced body weight and adipose tissue weight in diet-induced obese rats through a lipid-lowering action. Thus, it was a promising therapeutic agent for treatment of obesity. However, after repeated oral dosing of JTT-553 in rats and monkeys, plasma transaminase activities were increased in both animal

Correspondence: Hideaki Yokoyama (E-mail: hideaki.yokoyama@jt.com) 
H. Yokoyama et al.

species, although the magnitude of the change was mild and there were neither changes in other hepatic function parameters (e.g., alkaline phosphatase, lactate dehydrogenase and total bilirubin in blood) nor histopathological findings in the liver, suggesting no apparent hepatotoxicity (Yokoyama et al., 2018).

We previously reported that plasma transaminase activity was increased only when animals were fed after JTT-553 administration, and dietary fat mainly contributed to the increase in plasma transaminase activity (Yokoyama et al., 2018). We also found that transaminase activity was increased in the small intestine but not in the liver (Yokoyama et al., 2018). These results suggest that the increased plasma transaminase originates from the small intestine, where JTT-553 exhibits its pharmacological action, but not from the liver. However, the exact mechanism for the elevation of plasma and small intestinal transaminase activity by dietary fat under DGAT1inhibited conditions has not yet been elucidated.

Blood transaminases, namely alanine aminotransferase (ALT) and aspartate aminotransferase (AST), have been widely used as sensitive markers of tissue/organ damages, particularly hepatocellular damage, induced by drugs or diseases both in non-clinical toxicology studies and clinical studies (Ramaiah, 2007; Kobayashi et al., 2020). Meanwhile, ALT and AST are also known as key enzymes involved in amino acid and glucose metabolism (DeRosa and Swick, 1975). In addition, the activity of these transaminases is known to be altered by hormonal and nutritional modifications such as glucocorticoid treatment, feeding on a high-fat diet and dietary restriction (Rosen et al., 1959; Katchman and Zipf, 1970; Kobayashi et al., 2010, 2011). In non-clinical toxicology studies, blood transaminase levels are sometimes increased without the alteration of other hepatic function markers or histopathological changes in the liver suggesting hepatotoxicity (Kodama et al., 2008). Also in clinical trials, we sometimes encounter a phenomenon in which blood transaminase activity is slightly increased without the alteration of other hepatic function tests or any symptoms (Blane, 1987). Such an elevation of plasma transaminase activity sometimes makes it difficult to precisely assess the hepatotoxicity potential of new drug candidates.

Hepatotoxicity is becoming a significant public health issue because of its potential impact on not only the treatment of patients but also the development of new drugs (Kobayashi et al., 2020). Therefore, it is very important for new drug development to understand the mechanism of the elevations of blood transaminase activity without any other hepatotoxicity-associated findings. In this study, we have further investigated the mechanism for the
JTT-553-induced increase in blood transaminase activity. Especially, we sought to identify a lipid component responsible for the elevation using inhibitors for monoacylglycerol acyltransferase 2 (MGAT2) (Okuma et al., 2015) and intestinal microsomal triglyceride transfer protein (MTP) (Mera et al., 2011) as well as JTT-553 and fatty acids (FAs).

\section{MATERIALS AND METHODS}

\section{Chemicals}

JTT-553, which is a selective DGAT1 inhibitor (Tomimoto, et al., 2015a, 2015b), JTP-103237, 7-(4,6-ditert-butyl-pyrimidin-2-yl)-3-(4-tri-fluoromethoxy-phenyl)5,6,7,8-tetrahydro-[1,2,4]triazolo[4,3-a] pyrazine derivative, which is a selective MGAT2 inhibitor (Okuma, et al., 2015), and JTT-130, diethyl-2-(3-\{dimethylcarbamoyl4-[(4-trifluoromethylbiphenyl-2-carbonyl)amino]phenyl\} acetyloxymethyl)-2-phenylmalonate, which is a selective intestinal MTP inhibitor (Mera, et al., 2011), were synthesized in the Central Pharmaceutical Research Institute, Japan Tobacco Inc. (Osaka, Japan).

\section{Animals and experimental procedures}

Male F344/DuCrlCrlj rats were purchased from Charles River Japan Inc. (Kanagawa, Japan). Each experiment was initiated at their ages of 6 to 7 weeks old. Two to three rats were housed per stainless steel bracket cage with bedding in an air-conditioned animal room with a 12-hr light-dark cycle (lighting from 7:00 a.m. to 7:00 p.m.) at a temperature of $20-26^{\circ} \mathrm{C}$, a relative humidity of $30-70 \%$, and a ventilation rate of 10-20 times per hour. The rats were allowed free access to a commercial diet (CR-LPF, Oriental Yeast Co., Ltd., Tokyo, Japan). Tap water was available ad libitum. All animal experimental procedures were approved by the Institutional Animal Care and Use Committee of the Toxicology Research Laboratories, Central Pharmaceutical Research Institute, Japan Tobacco Inc. The experiments were conducted in accordance with the Japanese Law for the Humane Treatment and Management of Animals (Law No. 105, issued in October 1, 1973).

Blood was collected from the subclavian vein without anesthesia or from the abdominal aorta under isoflurane anesthesia, with lithium heparin-treated syringes. After the final sampling, all the animals were euthanized by exsanguination from the abdominal aorta under isoflurane anesthesia. Plasma was obtained by centrifugation $(7,300 \mathrm{~g}$, $4^{\circ} \mathrm{C}, 15 \mathrm{~min}$ ) and stored at $-80^{\circ} \mathrm{C}$ until use.

Plasma ALT and AST activities and plasma TAG concentrations were measured with a TBA-120FR auto- 
DGAT1 inhibition increases plasma transaminase via PKC pathway

mated analyzer (Canon Medical Systems Corporation, Tochigi, Japan) using standard reagents for ALT, AST and TAG (FUJIFILM Wako Pure Chemical Corporation, Osaka, Japan).

\section{Animal experiments}

Experiment 1: Time-course and dose-dependency of corn oil loading

JTT-553 $(30 \mathrm{mg} / \mathrm{kg})$ suspended in vehicle $[0.5 \%$ methylcellulose (Shin-Etsu Chemical Co., Ltd., Tokyo, Japan) aqueous solution $(0.5 \% \mathrm{MC})$ ] was administered once orally to rats (five animals per group) at $5.0 \mathrm{~mL} / \mathrm{kg}$ after overnight fasting. Corn oil (Sigma-Aldrich Co. LLC, St. Louis, MO, USA, $1 \mathrm{~g} / \mathrm{kg}$ ) was loaded orally to the animals at $1.1 \mathrm{~mL} / \mathrm{kg}$ at $0.5,1,2$ or $4 \mathrm{hr}$ after dosing JTT-553 or the vehicle. Blood was collected immediately before dosing JTT-553, and immediately before and at 2 , 4 and $6 \mathrm{hr}$ after corn oil loading to determine plasma ALT and AST activities and TAG concentrations.

Separately, JTT-553 $(30 \mathrm{mg} / \mathrm{kg})$ suspended in the vehicle $(0.5 \% \mathrm{MC})$ was administered once orally to rats (five animals per group) at $5.0 \mathrm{~mL} / \mathrm{kg}$ after overnight fasting. At $0.5 \mathrm{hr}$ after the dosing, corn oil $(0.3,1,3$ or $10 \mathrm{~g} / \mathrm{kg})$ was administered orally to the animals at $0.3,1.1,3.3$ or $11.1 \mathrm{~mL} / \mathrm{kg}$. The control animals were administered distilled water (DW, Otsuka Pharmaceutical Factory, Inc., Tokushima, Japan) at $11.1 \mathrm{~mL} / \mathrm{kg}$. Blood was collected immediately before dosing JTT-553, and immediately before and at 2, 4 and $6 \mathrm{hr}$ after the loading of corn oil or DW, and the plasma ALT and AST activities and plasma TAG concentrations were determined.

Experiment 2: Determination of transaminase $m R N A$ levels

JTT-553 $(30 \mathrm{mg} / \mathrm{kg})$ suspended in the vehicle $(0.5 \%$ $\mathrm{MC}$ ) or the vehicle alone was administered orally to rats (five animals per point) after overnight fasting at $5.0 \mathrm{~mL} / \mathrm{kg}$. At $4 \mathrm{hr}$ after the dosing, corn oil $(1 \mathrm{~g} / \mathrm{kg})$ or DW was administered orally to the animals at $1.1 \mathrm{~mL} / \mathrm{kg}$. At $0.5,1$ and $2 \mathrm{hr}$ after the loading of corn oil or DW, all the animals were euthanized by exsanguination from the abdominal aorta under isoflurane anesthesia and blood, the upper small intestine and the livers were collected. The upper small intestine was cut, opened and washed with saline. The mucosa was scraped off the small intestine with a glass slide. The aliquots of the samples of the small intestine and liver were transferred into RNAprotect Tissue Reagent (Qiagen N.V., Venlo, Netherland) and stored at $-80^{\circ} \mathrm{C}$ until use.

The small intestinal and hepatic mRNA levels of ALT1, ALT2, AST1, AST2 and $\beta$-actin were determined by quantitative reverse transcription polymerase chain reaction (qRT-PCR). Total RNA was isolated from the small intestine and liver samples using the Qiagen RNeasy Plus Mini kit (Qiagen N.V.) according to the manufacturer's instructions. cDNA was synthesized using the total RNA and SuperScript ${ }^{\mathrm{TM}}$ IV Reverse Transcriptase (Thermo Fisher Scientific, Waltham, MA, USA) according to the manufacturer's instructions. Real-time PCR was carried out with a QuantStudio 3 Real-Time PCR System (Thermo Fisher Scientific) using the diluted cDNA and TaqMan ${ }^{\mathrm{TM}}$ Gene Expression Master Mix with probes (ALT1, Rn00578989_g1; ALT2, Rn01538341_m1; AST1, Rn00561315_m1; AST2, Rn00820736_g1; $\beta$-actin, 4352931E) (Thermo Fisher Scientific) according to the manufacturer's instructions. The relative transaminase mRNA levels were determined following the normalization with $\beta$-actin mRNA levels and using the mixed tissue pool as the calibrator sample.

\section{Experiment 3: Effect of free FA-loading}

JTT-553 (30 mg/kg) suspended in vehicle (0.5\% MC) or the vehicle alone was administered once orally to rats (three animals per group) at $5.0 \mathrm{~mL} / \mathrm{kg}$ after overnight fasting. At $4 \mathrm{hr}$ after the dosing, oleic acid (Sigma-Aldrich), linoleic acid (Sigma-Aldrich) or corn oil was orally administered to the animals at $1 \mathrm{~g} / \mathrm{kg}(1.1 \mathrm{~mL} / \mathrm{kg})$. The control animals were administered DW at $1.1 \mathrm{~mL} / \mathrm{kg}$. Blood was collected immediately before dosing JTT-553 or the vehicle, and immediately before and at 2, 4 and $6 \mathrm{hr}$ after the loading of oleic acid, linoleic acid, corn oil or DW to determine their effects on plasma ALT activity.

\section{Experiment 4: Effects of a MGAT2 inhibitor and an intestinal MTP inhibitor}

The MGAT2 inhibitor, JTP-103237 (30 mg/kg) suspended in vehicle $(0.5 \% \mathrm{MC})$ or the vehicle alone was administered orally to rats (five animals per group) at $5.0 \mathrm{~mL} / \mathrm{kg}$ after overnight fasting. The dose level of JTP103237 was determined according to the previous report on its pharmacological study (Okuma, et al., 2015). At $1 \mathrm{hr}$ after the dosing, corn oil (1 g/ kg) or DW was loaded orally to the animals at $1.1 \mathrm{~mL} / \mathrm{kg}$. Blood was collected immediately before dosing JTP-103237 or the vehicle, and immediately before and at 2, 4 and $6 \mathrm{hr}$ after the loading of corn oil or DW, and plasma ALT activity was determined.

Separately, the intestinal MTP inhibitor, JTT-130 $(10 \mathrm{mg} / \mathrm{kg})$ dissolved in vehicle [polyethylene glycol 400 (PEG400; Toho Yakuhin Co., Ltd., Tokyo, Japan)] or the vehicle alone was administered orally to rats (five animals per group) at $2.0 \mathrm{~mL} / \mathrm{kg}$ after overnight fasting. The dose level of JTT-130 was determined according to 
H. Yokoyama et al.

the previous report on its pharmacological study (Mera, et al., 2011). At $0.5 \mathrm{hr}$ after the dosing, corn oil $(1 \mathrm{~g} / \mathrm{kg})$ or DW was administered orally to the animals at $1.1 \mathrm{~mL} /$ $\mathrm{kg}$. Blood was collected immediately before dosing JTT130 or vehicle, and immediately before and at 2, 4 and $6 \mathrm{hr}$ after the loading of corn oil or DW, and plasma ALT activity was determined.

\section{Experiment 5: Effect on protein kinase C (PKC) activity in the small intestine}

JTT-553 $(30 \mathrm{mg} / \mathrm{kg})$ suspended in the vehicle $(0.5 \%$ $\mathrm{MC})$ or the vehicle alone was administered orally to rats (five animals per group) after overnight fasting at $5.0 \mathrm{~mL} / \mathrm{kg}$. At $4 \mathrm{hr}$ after the dosing, corn oil $(1 \mathrm{~g} / \mathrm{kg})$ or DW was administered orally to the animals at $1.1 \mathrm{~mL} / \mathrm{kg}$. At $2 \mathrm{hr}$ after the loading of corn oil or DW, all the animals were euthanized by exsanguination from the abdominal aorta under isoflurane anesthesia and the middle small intestines were collected. The middle small intestines were cut, opened and washed with saline. The mucosa was scraped off the small intestine with a glass slide. The aliquots of the small intestine samples were frozen with liquid nitrogen and stored at $-80^{\circ} \mathrm{C}$ until use.

Total protein was extracted from the small intestine samples using T-PER ${ }^{\mathrm{TM}}$ Tissue Protein Extraction Reagent (Thermo Fisher Scientific) with Halt Protease Inhibitor Single-Use Cocktail (Thermo Fisher Scientific). PKC activity was measured using the total protein extract and PKC Kinase Activity Kit (Enzo Life Sciences Inc., Farmingdale, NY, USA). The protein concentration was quantified with Pierce ${ }^{\mathrm{TM}}$ BCA Protein Assay Kit (Thermo Fisher Scientific). All procedures were according to the manufacturer's instructions for each reagent/kit. The PKC activity was normalized with $\mathrm{mg}$ total protein levels and expressed as relative activity.

\section{Experiment 6: Effect of a pan-PKC inhibitor}

JTT-553 $(30 \mathrm{mg} / \mathrm{kg})$ suspended in vehicle $(0.5 \% \mathrm{MC})$ or the vehicle alone was administered once orally to rats (five animals per group) after overnight fasting. At $3.5 \mathrm{hr}$ after the dosing, the pan-PKC inhibitor, sotrastaurin (30 mg/kg; Chemscene LLC., Monmouth Junction, NJ, USA) suspended in vehicle [20\% (v/v) PEG400 aqueous solution $(20 \%$ PEG400)] or the vehicle alone was orally administered to the animals at $10 \mathrm{~mL} / \mathrm{kg}$. At $0.5 \mathrm{hr}$ after dosing sotrastaurin or the vehicle, corn oil $(1 \mathrm{~g} / \mathrm{kg})$ was loaded orally to the animals at $1.1 \mathrm{~mL} / \mathrm{kg}$. Blood was collected at $2 \mathrm{hr}$ after corn oil loading, and plasma ALT activity was determined.

\section{Statistical analysis}

The mean values and standard deviations (SDs) in each group were calculated for the plasma ALT and AST activities, plasma TAG concentrations, relative transaminase (ALT1, ALT2, AST1 and AST2) mRNA levels and small intestinal PKC activity. Unpaired Student's $t$-test and Dunnett's test were performed using Microsoft Excel (Microsoft Corporation, Tokyo, Japan) and Pharmaco Basic (Scientist Press Co. Ltd., Tokyo, Japan), respectively. The levels of significance were set at 5\% (one-tailed).

\section{RESULTS}

\section{Effects of the loading timing and volume of corn oil on the JTT-553-dependent transaminase increases}

We first investigated whether the elevation of plasma transaminase activity was related to the pharmacological action of JTT-553 (i.e., inhibition of lipid absorption in the small intestine) by analyzing the association between corn oil loading conditions (timing and volume) and the extent of increase in plasma transaminase activity.

To investigate the effects of corn oil-loading timing on the plasma transaminase activity in rats treated with JTT-553, corn oil was loaded to rats at $0.5,1,2$ or $4 \mathrm{hr}$ after JTT-553 dosing. Under any of the conditions, plasma ALT and AST activities increased most in rats loaded with corn oil at $4 \mathrm{hr}$ after the loading by $38 \%$ to $54 \%$ and $12 \%$ to $19 \%$, respectively, when compared with the pre-loading values (Fig. 1A). The changes in the timing of corn oil loading did not affect the plasma TAG concentrations (Fig. 1A).

We then changed the corn oil volume to investigate its effects on the plasma transaminase activity in JTT553-treated rats (Fig. 1B). Corn oil was loaded orally to rats at $0.3,1,3$ or $10 \mathrm{~g} / \mathrm{kg}$ at $0.5 \mathrm{hr}$ after JTT-553 dosing. While $0.3 \mathrm{~g} / \mathrm{kg}$ corn oil had no effects on the plasma ALT and AST activities, corn oil at $1 \mathrm{~g} / \mathrm{kg}$ and above increased the transaminase activities from $2 \mathrm{hr}$ after corn oil loading. At $6 \mathrm{hr}$ after the loading, ALT and AST activities were increased by 69 to $135 \%$ and 12 to $24 \%$, respectively, when compared with the control group. The magnitude of the increase was comparable at 1,3 and $10 \mathrm{~g} / \mathrm{kg}$. Although the plasma TAG concentration at $6 \mathrm{hr}$ after corn oil loading of the $3 \mathrm{~g} / \mathrm{kg}$ group was statistically higher than that of the control group, no dose-dependent changes in TAG concentrations were observed.

\section{Transaminase mRNA levels in the small intestine}

The results suggested that the elevation of the plasma transaminase activity after JTT-553 dosing in combi- 
DGAT1 inhibition increases plasma transaminase via PKC pathway
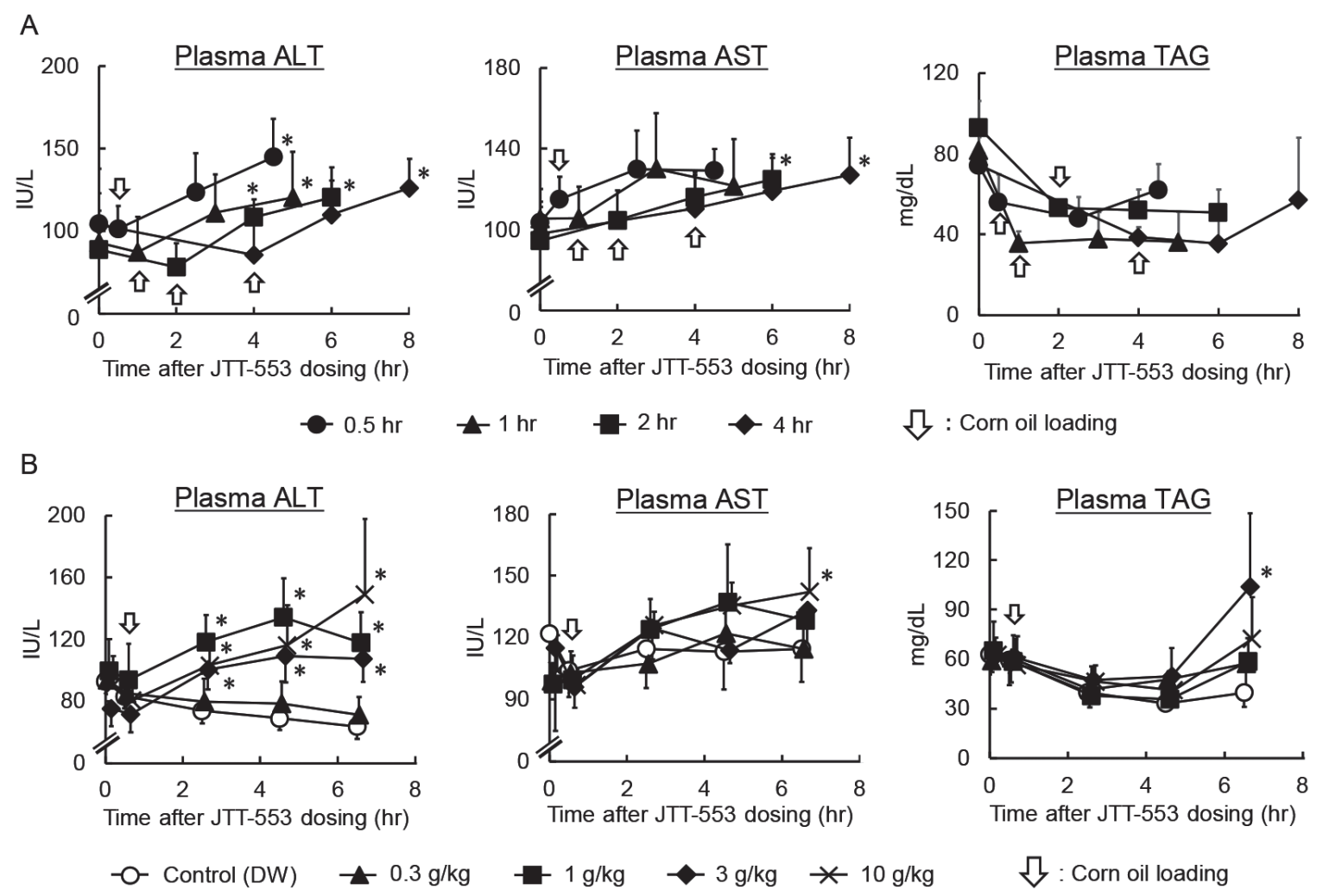

: Corn oil loading
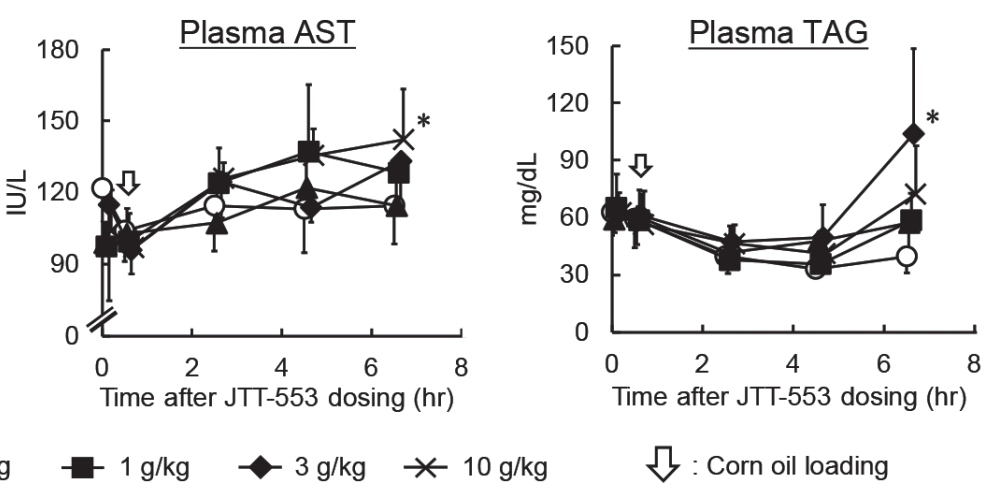

Fig. 1. Effects of the loading timing and volume of corn oil on the JTT-553-dependent transaminase increases. JTT-553 (30 mg/kg) was administered orally to fasted rats. (A) At $0.5,1,2$ or $4 \mathrm{hr}$ after dosing JTT-553, corn oil (1 g/kg) was loaded orally to the animals. (B) At $0.5 \mathrm{hr}$ after JTT-553 dosing, corn oil $(0.3,1,3$ or $10 \mathrm{~g} / \mathrm{kg}$ ) or DW was loaded orally to the animals. (A, B) Blood was collected immediately before JTT-553 dosing, and immediately before and at 2, 4 and 6 hr after the loading of corn oil or DW. Plasma ALT and AST activities and plasma TAG concentrations were measured as described in the Materials and Methods section. Data are shown as mean \pm S.D. $(n=5){ }^{*}, p<0.05$; significantly different from the values immediately before corn oil loading (A) or the control groups (B) (Dunnett's test).

nation with corn oil was related to the pharmacological action of JTT-553. To investigate the effects of DGAT1 inhibition and corn oil loading on transaminase mRNA levels in rat small intestines and livers, JTT-553 or the vehicle alone was administered once orally to rats in combination with corn oil or DW. Under these conditions, plasma TAG concentrations in the vehicle control group increased from $1 \mathrm{hr}$ after corn oil loading (e.g., twofold at $2 \mathrm{hr}$ after the loading when compared with the vehicle control group loaded with DW, Fig. 2A). In contrast, in the JTT-553-treated group, plasma TAG concentration did not change after corn oil loading, as expected. In the JTT-553-treated group loaded with corn oil, plasma ALT activity increased from $1 \mathrm{hr}$ after corn oil loading (by $93 \%$ at $2 \mathrm{hr}$ after the loading when compared with the vehicle control group loaded with DW, Fig. 2A). Plasma AST activity also increased at $2 \mathrm{hr}$ after corn oil loading (by $12 \%$ when compared with the vehicle control group loaded with DW, Fig. 2A). In the vehicle-treated rats loaded with corn oil and JTT-553-treated rats loaded with DW, plasma ALT and AST activities did not increase.

As shown in Fig. 2B, intestinal mRNA levels of ALT1, ALT2, AST1 and AST2 did not increase up to $2 \mathrm{hr}$ after corn oil loading in the JTT-553-treated rats loaded with corn oil when compared with the vehicle control group loaded with DW although intestinal ALT2, AST1 or AST2 mRNA levels decreased significantly at 0.5 or $2 \mathrm{hr}$ after corn oil loading. Although a reason for the decreases remains unclear, considering the direction of the changes, the changes are unlikely to be the cause of the elevation of plasma transaminase activity. In addition, those mRNA levels in the liver also did not change at $1 \mathrm{hr}$ after corn oil loading. In the vehicle-treated rats loaded with corn oil and JTT-553-treated rats loaded with DW, the mRNA lev- 
H. Yokoyama et al.
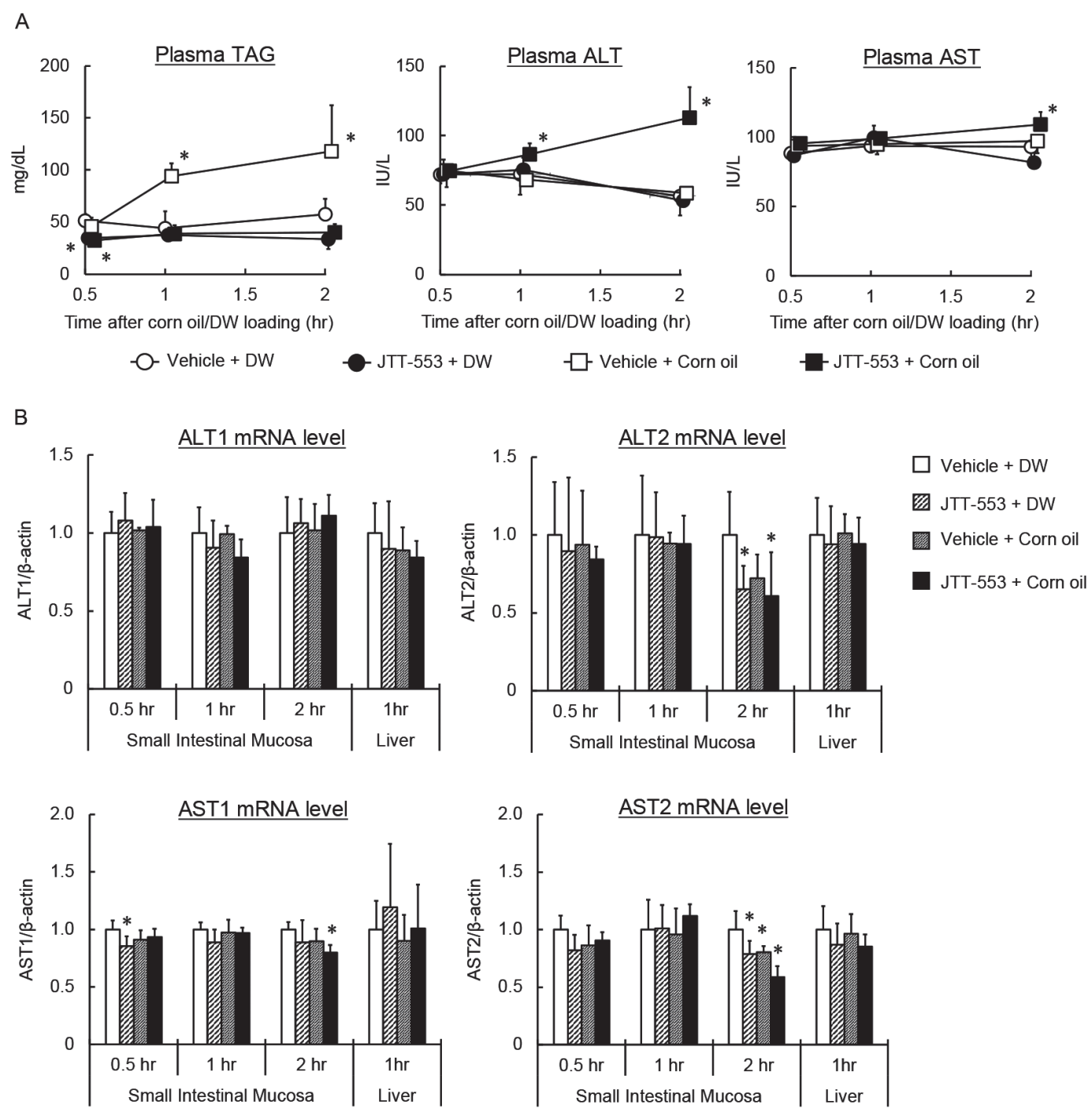

Fig. 2. Effects of DGAT1 inhibition and corn oil loading on transaminase mRNA levels in rat small intestines and livers. JTT-553 $(30 \mathrm{mg} / \mathrm{kg})$ or the vehicle $(0.5 \% \mathrm{MC})$ alone was orally administered once to fasted rats, and $4 \mathrm{hr}$ later, corn oil ( $1 \mathrm{~g} / \mathrm{kg})$ or DW was loaded orally to the animals. Blood, small intestines and livers were collected $0.5,1$ and $2 \mathrm{hr}$ after the loading of corn oil or DW. Plasma TAG concentrations, plasma ALT and AST activities (A) and small intestinal and hepatic mRNA levels of ALT1, ALT2, AST1 and AST2 (B) were measured as described in the Materials and Methods section. Data are shown as mean \pm S.D. $(n=5)^{*}, p<0.05$; significantly different from the vehicle control rats loaded with DW (Dunnett's test).

els in the intestine and liver also showed no increase.

\section{Effects of free FA-loading}

Since JTT-553 inhibits the DGAT1-mediated re-synthesis of TAG from a fatty acyl-CoA and DAG in the small intestine, TAG components, i.e., fatty acyl-CoA, MAG and DAG, were expected to accumulate in the enterocytes after corn oil loading. To clarify the involvement of these lipid components in the elevation of plas- ma transaminase activity, we first investigated the effects of FA loading instead of corn oil on plasma transaminase activity.

Oleic acid or linoleic acid, which are highly present in corn oil (United States Department of Agriculture (USDA), 2017-2018), was loaded orally to rats at $1 \mathrm{~g} / \mathrm{kg}$ $4 \mathrm{hr}$ after JTT-553 dosing. Plasma ALT activity was significantly increased by corn oil loading in the JTT553 -treated groups (Fig. 3). However, such an increase 
DGAT1 inhibition increases plasma transaminase via PKC pathway

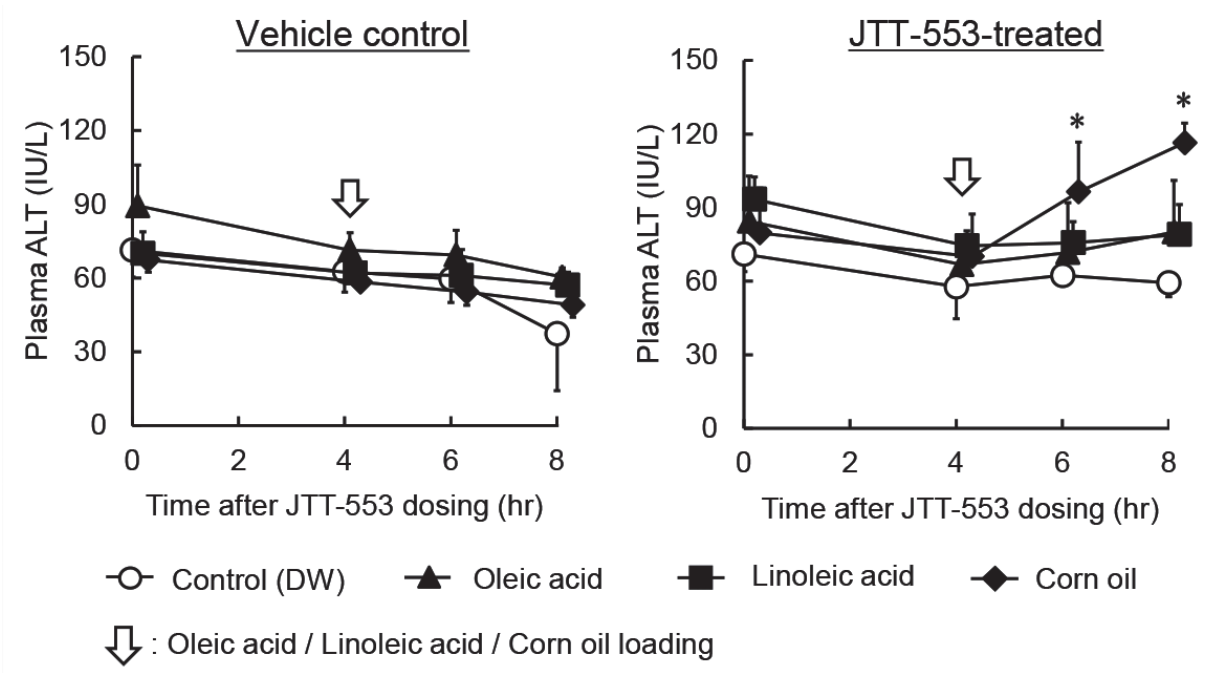

Fig. 3. Effects of free FA loading on plasma ALT activity in the JTT-553-treated rats. JTT-553 (30 mg/kg) or the vehicle $(0.5 \%$ $\mathrm{MC})$ alone was administered orally to fasted rats, and $4 \mathrm{hr}$ later, oleic acid, linoleic acid, corn oil (1 g/ $/ \mathrm{kg})$ or DW was loaded orally to the animals. Blood was collected immediately before JTT-553 dosing, and immediately before and at 2, $4 \mathrm{and} 6 \mathrm{hr}$ after the loading of oleic acid, linoleic acid, corn oil or DW. Plasma ALT activity was measured as described in the Materials and Methods section. Data are shown as mean \pm S.D. $(\mathrm{n}=3){ }^{*}, p<0.05$; significantly different from the control groups (Dunnett's test).

was observed in neither oleic acid- nor linoleic acid-loaded rats (Fig. 3).

\section{Effects of an MGAT2 inhibitor and an intestinal MTP inhibitor}

Plasma ALT activity was not increased by FA loading in rats treated with a DGAT1 inhibitor. We next investigated whether MAG or TAG was responsible for the elevation of the plasma transaminase activity using a MGAT2 inhibitor (JTP-103237) or an intestinal MTP inhibitor (JTT-130). Rats were administered these inhibitors orally and then corn oil was loaded. In contrast to JTT-553, pretreatment with JTP-103237 or JTT-130 did not induce the corn oil loading-mediated increases in plasma ALT activity at any sampling point (Fig. 4). Although plasma ALT activity was statistically higher in rats treated with JTT-130 and DW than those in rats treated with the vehicle and DW at some time points, the changes were considered to be incidental based on the ALT activity of rats treated with the vehicle and DW in other experiments (unpublished data).

\section{PKC activity in the small intestine}

The elevation of the plasma transaminase activity was observed after corn oil but not FA loading in rats treated with the DGAT1 inhibitor and treatment with an MGAT2 inhibitor or an intestinal MTP inhibitor did not induce the corn oil loading-mediated elevation of transaminase activities. These results suggested that DAG could contribute to the elevation. Since DAG is well known to activate classic/novel PKCs (Yang and Kazanietz, 2003; Newton, 2001), we investigated the effects of DGAT1 inhibition and corn oil loading on PKC activity in the small intestine. JTT-553 or the vehicle alone was administered once orally to rats in combination with corn oil or DW. Under these conditions, small intestinal PKC activity in the JTT553 -treated rats loaded with corn oil increased at $2 \mathrm{hr}$ after corn oil loading (by $49 \%$ when compared with the vehicle control group loaded with DW, Fig. 5).

\section{Effects of a pan-PKC inhibitor}

DGAT1 inhibition and corn oil loading increased PKC activity in the small intestine of rats. To clarify the involvement of the small intestinal PKC activation in the elevation of plasma transaminase activity, we investigated the effects of treatment with the pan-PKC inhibitor sotrastaurin on plasma transaminase activity. Rats were administered the inhibitor orally at $3.5 \mathrm{hr}$ after JTT553 dosing then with corn oil at $0.5 \mathrm{hr}$ after sotrastaurin administration. Under these conditions, JTT-553 and corn oil treatment increased plasma ALT activity by $69 \%$, and sotrastaurin pretreatment significantly prevented the increase, as we expected (Fig. 6). No significant increase in ALT activity was observed after corn oil loading in the 
H. Yokoyama et al.

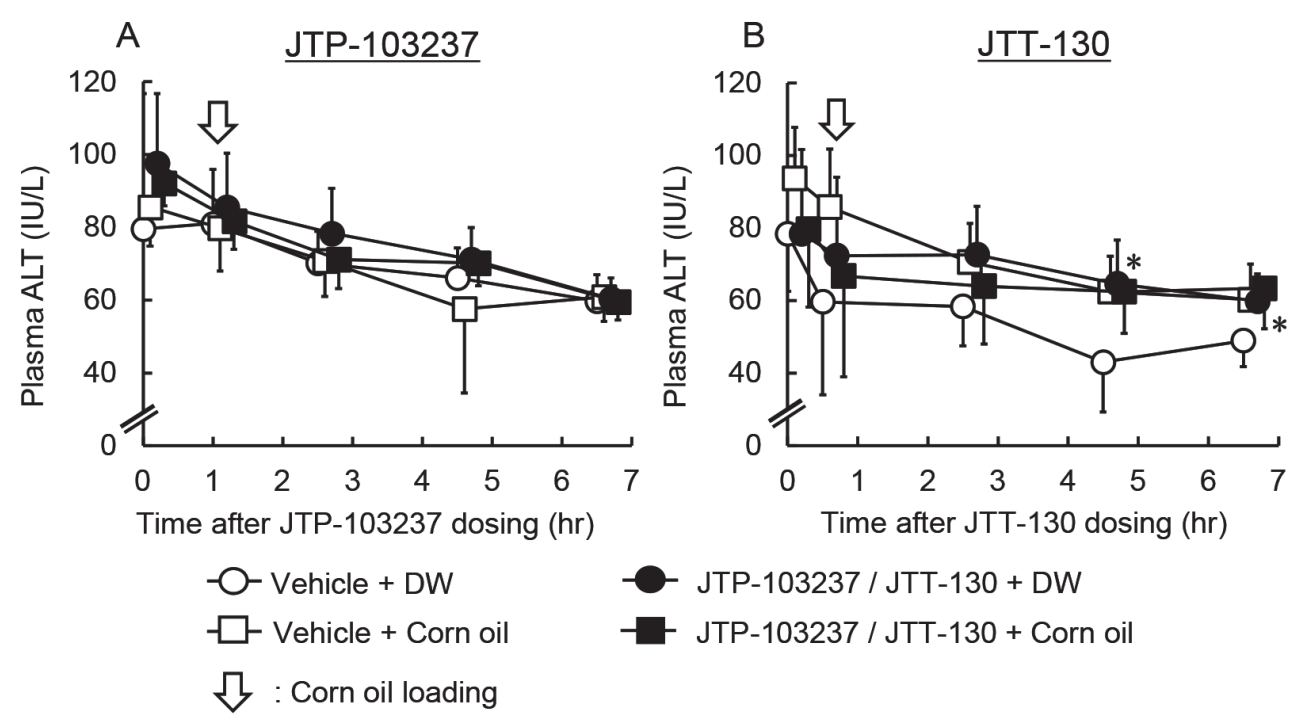

Fig. 4. Effects of the MGAT2 inhibitor JTP-103237 and the intestinal MTP inhibitor JTT-130 on plasma ALT activity. (A) JTP$103237(30 \mathrm{mg} / \mathrm{kg})$ or the vehicle $(0.5 \% \mathrm{MC})$ alone was administered orally to fasted rats, and $1 \mathrm{hr}$ later, corn oil (1 g/kg) or DW was loaded orally to the animals. (B) JTT-130 $(10 \mathrm{mg} / \mathrm{kg})$ or the vehicle (PEG400) alone was administered orally to rats, and $0.5 \mathrm{hr}$ later, corn oil $(1 \mathrm{~g} / \mathrm{kg})$ or DW was loaded orally to the animals. Blood was collected immediately before JTP-103237 or JTT-130 dosing, and immediately before and at 2, 4 and $6 \mathrm{hr}$ after the loading of corn oil or DW. (A, B) Plasma ALT activity was measured as described in the Materials and Methods section. Data are shown as mean \pm S.D. $(n=5)$. *, $p<0.05$; significantly different from the vehicle control groups loaded with DW (Dunnett's test).

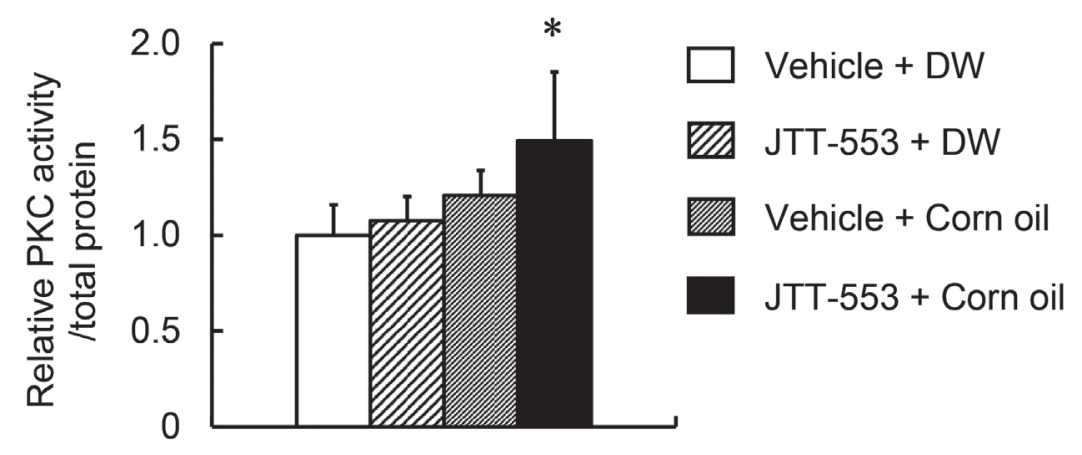

Fig. 5. Effects of DGAT1 inhibition and corn oil loading on PKC activity in rat small intestines. JTT-553 (30 mg/kg) or the vehicle $(0.5 \% \mathrm{MC})$ alone was orally administered once to fasted rats, and $4 \mathrm{hr}$ later, corn oil $(1 \mathrm{~g} / \mathrm{kg})$ or DW was loaded orally to the animals. Small intestines were collected $2 \mathrm{hr}$ after the loading of corn oil or DW. Small intestinal PKC activity was measured as described in the Materials and Methods section. Data are shown as mean \pm S.D. $(\mathrm{n}=5) *, p<0.05$; significantly different from the vehicle control rats loaded with DW (Dunnett's test).

sotrastaurin-pretreated group without JTT-553 (Fig. 6, open bars).

\section{DISCUSSION}

In this study, we investigated the mechanism for the increase in blood transaminase activity by treatment with the DGAT1 inhibitor JTT-553 and corn oil. We found that the elevation occurred only during the pharmacologically active period of the DGAT1 inhibitor and the magnitude did not depend on the corn oil volume. The transaminase mRNA levels in the small intestine as well as the liver did not increase after the DGAT1 inhibitor treatment and corn oil loading. In addition, the JTT-553-dependent elevation was not observed after oleic acid or linoleic acid loading, and treatment with the MGAT2 inhibitor 
DGAT1 inhibition increases plasma transaminase via PKC pathway

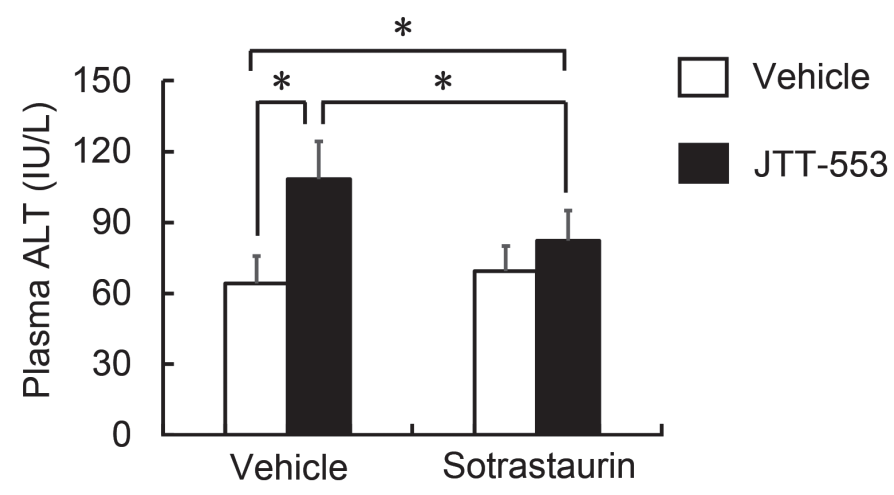

Fig. 6. Effects of the pan-PKC inhibitor sotrastaurin on plasma ALT activity in the JTT-553-treated rats. JTT-553 (30 mg/kg) or the vehicle $(0.5 \% \mathrm{MC})$ alone was administered orally to fasted rats, $3.5 \mathrm{hr}$ later, sotrastaurin $(30 \mathrm{mg} / \mathrm{kg})$ or the vehicle $(20 \%$ PEG400) alone was administered orally to the animals, and $0.5 \mathrm{hr}$ later, corn oil (1 g/ kg) was loaded orally to the animals. Blood was collected $2 \mathrm{hr}$ after the corn oil loading. Plasma ALT activity was measured as described in the Materials and Methods section. Data are shown as mean \pm S.D. $(\mathrm{n}=5) .{ }^{*}, p<0.05$; significantly different from the vehicle $(0.5 \% \mathrm{MC})$ control rats pretreated with the vehicle of sotrastaurin (20\% PEG400) or the JTT-553-treated rats pretreated with sotrastaurin (Dunnett's test).

JTP-103237 or the intestinal MTP inhibitor JTT-130 did not induce the elevation in contrast to JTT-553. Finally, small intestinal PKC activity increased after treatment with the DGAT1 inhibitor and corn oil, and the elevation of ALT was suppressed by pretreatment with the pan-PKC inhibitor sotrastaurin. Although the induction of transaminase expression was reported to contribute to the elevation of plasma transaminase activity in some cases (Pappas, 1986; Kobayashi et al., 2010, 2011), our present results strongly suggest that the elevation associated with the DGAT1 inhibitor does not result from the increased expression of transaminase in the small intestine, but the DGAT1 inhibitor treatment accumulates DAG in enterocytes by its pharmacological actions and increases plasma transaminase activity via the activation of the DAG-PKC pathway in rats.

Plasma transaminase activity increased after corn oil loading between 0.5 and $4 \mathrm{hr}$ after dosing the DGAT1 inhibitor (Fig. 1A) while the elevation of plasma TAG level after corn oil loading was suppressed by the DGAT1 inhibitor (Fig. 1A). Since the time to reach the maximum plasma concentration of JTT-553 was $0.5 \mathrm{hr}$ after its dosing (data not shown), the corn oil loading was considered not to affect the systemic exposure to JTT-553. The magnitude of the elevation of plasma transaminase activity was not dependent on the doses of corn oil between 1 to $10 \mathrm{~g} / \mathrm{kg}$ (Fig. 1B). Plasma transaminase activity did not increase by loading corn oil at the load level of 0 or $0.3 \mathrm{~g} / \mathrm{kg}$ after dosing the DGAT1 inhibitor or by only corn oil loading (Figs. 1B and 2A). We previously reported that the elevation of plasma transaminase activity noted in oral repeated-dose toxicity studies in rats and monkeys for the DGAT1 inhibitor was not dose-dependent and not accompanied by any change in other hepatic function parameters or any hepatotoxicity-associated histopathological findings. In addition, the origin of the increased transaminase in the plasma was the small intestine where the DGAT1 inhibitor exhibits its pharmacological action (Yokoyama et al., 2018). Based on these results, the elevation of plasma transaminase activity after treatment with the DGAT1 inhibitor is probably related to the pharmacological action of the DGAT1 inhibitor, i.e., suppression of plasma TAG level elevation after corn oil loading.

Plasma ALT activity did not change when oleic acid or linoleic acid was loaded instead of corn oil (Fig. 3). Since oleic acid or linoleic acid was loaded with the DGAT1 inhibitor, the re-synthesis of TAG from a fatty acyl-CoA and DAG was probably inhibited, and fatty acyl-CoA derived from the FA could be accumulated in enterocytes. These results suggest that FAs are not responsible for the elevation of plasma transaminase activity associated with DGAT1 inhibition.

We also demonstrate that the transaminase activity did not change after corn oil loading in the MGAT2 inhibitortreated rats (Fig. 4A). MGAT is an enzyme that catalyzes the first step of TAG synthesis, the re-synthesis of DAG from a fatty acyl-CoA and MAG in the small intestine. JTP-103237 selectively inhibits MGAT2 (Okuma et al., 2015), an isozyme that is highly expressed in the intestine of both humans and rodents (Yen and Farese, 2003). 
H. Yokoyama et al.

Okuma et al. reported that the MGAT2 inhibitor significantly increased the contents of MGAT substrates, namely MAG and FAs, in the small intestine after loading a lipid emulsion (Okuma et al., 2015). Taken together, these results indicate that MAG and FAs in enterocytes are not responsible for the elevation of plasma transaminase activity.

Similarly, treatment with the MTP inhibitor in combination with corn oil did not alter plasma ALT activity (Fig. 4B). In enterocytes and hepatocytes, MTP is localized in the endoplasmic reticulum and plays an important role in the synthesis of chylomicron and the transfer of TAG and cholesteryl ester (Jamil et al., 1995). JTT-130 used as an intestinal MTP inhibitor was designed to be rapidly hydrolyzed to an inactive metabolite after absorption from the small intestine and to inhibit intestinal MTP specifically. This MTP inhibitor was reported to suppress an increase in ${ }^{14} \mathrm{C}$ radioactivity in blood as well as in the upper small intestine and the intestinal luminal content after $\left[{ }^{14} \mathrm{C}\right]$ triolein loading (Mera et al., 2011). It was thus expected that TAG was retained in the enterocytes after corn oil loading in rats pretreated with the MTP inhibitor. Taken together, our present results suggest that TAG in the enterocytes is not responsible for the elevation of plasma transaminase activity induced by the DGAT1 inhibitor.

Taken together, the results obtained raised a possibility that DAG contributed to the elevation of plasma transaminase activity. DAG binds to the $\mathrm{C} 1$ domain of two subgroups of the PKC family, namely classic PKCs (PKC $\alpha$, $\beta 1, \beta 2$ and $\gamma$ ) and novel PKCs (PKC $\delta, \varepsilon, \eta$ and $\theta$ ), to activate them (Yang and Kazanietz, 2003; Newton, 2001). We thus investigated PKC activity in the small intestine and found that the small intestinal PKC activity was increased by treatment with the DGAT1 inhibitor and corn oil (Fig. 5). In addition, we investigated the involvement of PKCs in the elevation of plasma transaminase activity by treating rats with a pan-PKC inhibitor (sotrastaurin) in combination with the DGAT1 inhibitor before corn oil loading. As we anticipated, the ALT elevation was suppressed by pretreatment with sotrastaurin (Fig. 6), suggesting possible involvement of PKCs in the DGAT1 inhibitor-associated transaminase elevation.

At this moment, it remains unclear how PKC activation in enterocytes increases plasma transaminase activity. PKCs are a family of serine/threonine kinases involved in various cellular processes, including apoptosis, proliferation, differentiation and cell survival (Kolczynska et al., 2020; Yang and Kazanietz, 2003). Treatment with inducers of hepatocyte apoptosis is known to increase plasma ALT activity in mice (Bajt et al., 2000; McGill, 2016). Additionally, PKC has been reported to change the permeability of the intestinal barrier (Clarke et al., 2000; Ulluwishewa et al., 2011) and a chemical-related activation of PKCs in intestinal epithelial cells possibly strengthens the integrity of intestinal tight junction barrier (Jo et al., 2017; Cario et al., 2004). Based on these reports, DAG in enterocytes might alter the plasma transaminase activity through apoptotic signaling leading to transaminase leakage and/or changing the permeability of the intestinal barrier via PKC activation although there were no histopathological findings in the small intestine, except for lipid-like vacuoles in the jejunum, after one-month repeated oral dosing of the DGAT1 inhibitor in rats and monkeys (Yokoyama et al., 2018).

We previously reported that the elevation of plasma transaminase activity was also suppressed by pretreatment with the transcription inhibitor actinomycin D (Yokoyama et al., 2018). Actinomycin D was reported to decrease PKC contents in neuroblastoma cells (Miñana et al., 1991). Additionally, actinomycin D blocks the transcriptional alterations induced by $\mathrm{PKC}$ activation in intestinal epithelial cells (Pysz et al., 2014; Pan and Stevens, 1995). These facts and our present results also suggest that DAG synthesized in enterocytes after corn oil loading with the DGAT1 inhibitor activates classic/novel PKCs and PKC-dependent cellular signals increase plasma transaminase activity through an as-yet-unknown mechanism (Fig. 7).

\section{ACKNOWLEDGMENTS}

The authors would like to thank the invaluable suggestions and technical supports from Dr. Toshiyuki Shoda, Dr. Akio Kobayashi, Dr. Shoichiro Sugai and the staff in the Toxicology Research Laboratories, Central Pharmaceutical Research Institute, JAPAN TOBACCO INC.

Conflict of interest---- The authors declare that there is no conflict of interest. 
DGAT1 inhibition increases plasma transaminase via PKC pathway

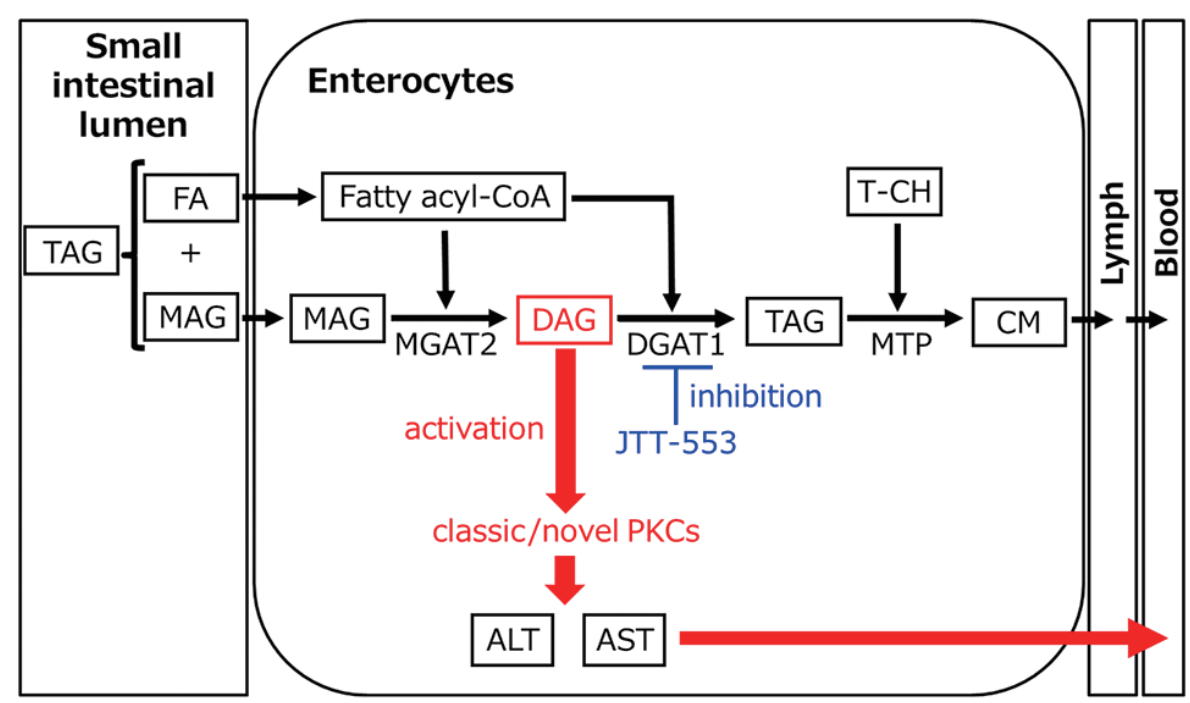

Fig. 7. Pharmacological action of JTT-553 and a proposed mechanism for the elevation of plasma transaminase activity.

\section{REFERENCES}

Bajt, M.L., Lawson, J.A., Vonderfecht, S.L., Gujral, J.S. and Jaeschke, H. (2000): Protection against Fas receptor-mediated apoptosis in hepatocytes and nonparenchymal cells by a caspase- 8 inhibitor in vivo: evidence for a postmitochondrial processing of caspase-8. Toxicol. Sci., 58, 109-117.

Blane, G.F. (1987): Comparative toxicity and safety profile of fenofibrate and other fibric acid derivatives. Am. J. Med., 83 (5B), 26-36.

Cario, E., Gerken, G. and Podolsky, D.K. (2004): Toll-like receptor 2 enhances ZO-1-associated intestinal epithelial barrier integrity via protein kinase C. Gastroenterology, 127, 224-238.

Cases, S., Smith, S.J., Zheng, Y.W., Myers, H.M., Lear, S.R., Sande, E., Novak, S., Collins, C., Welch, C.B., Lusis, A.J., Erickson, S.K. and Farese, R.V. Jr. (1998): Identification of a gene encoding an acyl CoA:diacylglycerol acyltransferase, a key enzyme in triacylglycerol synthesis. Proc. Natl. Acad. Sci. USA, 95, 13018-13023.

Cases, S., Stone, S.J., Zhou, P., Yen, E., Tow, B., Lardizabal, K.D., Voelker, T. and Farese, R.V. Jr. (2001): Cloning of DGAT2, a second mammalian diacylglycerol acyltransferase, and related family members. J. Biol. Chem., 276, 38870-38876.

Clarke, H., Marano, C.W., Peralta Soler, A. and Mullin, J.M. (2000): Modification of tight junction function by protein kinase C isoforms. Adv. Drug Deliv. Rev., 41, 283-301.

DeRosa, G. and Swick, R.W. (1975): Metabolic implications of the distribution of the alanine aminotransferase isoenzymes. J. Biol. Chem., 250, 7961-7967.

Jamil, H., Dickson, J.K. Jr., Chu, C.H., Lago, M.W., Rinehart, J.K., Biller, S.A., Gregg, R.E. and Wetterau, J.R. (1995): Microsomal triglyceride transfer protein. Specificity of lipid binding and transport. J. Biol. Chem., 270, 6549-6554.

Jo, H., Hwang, D., Kim, J.K. and Lim, Y.H. (2017): Oxyresveratrol improves tight junction integrity through the PKC and MAPK signaling pathways in Caco-2 cells. Food Chem. Toxicol., 108 (Pt A), 203-213.
Katchman, B.J. and Zipf, R.E. (1970): Correlation between triglycerides and glutamic-pyruvic transaminase in men on high-fat diets. Clin. Chem., 16, 118-123.

Kobayashi, A., Oshida, S., Yamazaki, Y., Maekawa, T., Kuno, H., Sugai, S., Sakakibara, H. and Shimoi, K. (2010): Relationships between plasma and tissue transaminase activities in rats maintained under different feeding conditions. J. Toxicol. Sci., 35, 639-652.

Kobayashi, A., Yokoyama, H., Kataoka, J., Ishida, T., Kuno, H., Sugai, S., Sakakibara, H. and Shimoi, K. (2011): Effects of spaced feeding on gene expression of hepatic transaminase and gluconeogenic enzymes in rats. J. Toxicol. Sci., 36, 325-337.

Kobayashi, A., Suzuki, Y. and Sugai, S. (2020): Specificity of transaminase activities in the prediction of drug-induced hepatotoxicity. J. Toxicol. Sci., 45, 515-537.

Kodama, T., Watanabe, E., Tsubuku, S., Otabe, A., Mochizuki, M., Masuyama, T. and Bernard, B.K. (2008): Studies of the toxicological potential of capsinoids: VII. A 13-week toxicity study of dihydrocapsiate in rats. Int. J. Toxicol., 27 (Suppl 3), 79-100.

Kolczynska, K., Loza-Valdes, A., Hawro, I. and Sumara, G. (2020): Diacylglycerol-evoked activation of PKC and PKD isoforms in regulation of glucose and lipid metabolism: a review. Lipids Health Dis., 19, 113.

McGill, M.R. (2016): The past and present of serum aminotransferases and the future of liver injury biomarkers. EXCLI J., 15, 817-828.

Mera, Y., Odani, N., Kawai, T., Hata, T., Suzuki, M., Hagiwara, A., Katsushima, T. and Kakutani, M. (2011): Pharmacological characterization of diethyl-2-(3-dimethylcarbamoyl4 - [( 4 '-trifluoromethylbipheny $1-2$-carbonyl)amino] phenylacetyloxymethyl)-2-phenylmalonate (JTT-130), an intestine-specific inhibitor of microsomal triglyceride transfer protein. J. Pharmacol. Exp. Ther., 336, 321-327.

Miñana, M.D., Felipo, V. and Grisolía, S. (1991): Actinomycin D decreases protein kinase $\mathrm{C}$ content and induces neuritogenesis in neuroblastoma cells. FEBS Lett., 280, 245-246. 


\section{H. Yokoyama et al.}

Newton, A.C. (2001): Protein kinase C: structural and spatial regulation by phosphorylation, cofactors, and macromolecular interactions. Chem. Rev., 101, 2353-2364.

Okuma, C., Ohta, T., Tadaki, H., Hamada, H., Oda, T., Taniuchi, H., Yamanaka, K., Ishii, Y., Ohe, Y., Yata, S., Nishiu, J., Aratsu, Y., Oshida, S., Kume, S. and Kakutani, M. (2015): JTP-103237, a novel monoacylglycerol acyltransferase inhibitor, modulates fat absorption and prevents diet-induced obesity. Eur. J. Pharmacol., 758, 72-81.

Pan, M. and Stevens, B.R. (1995): Differentiation- and protein kinase C-dependent regulation of alanine transport via system B. J. Biol. Chem., 270, 3582-3587.

Pappas, N.J. Jr. (1986): Source of increased serum aspartate and alanine aminotransferase: cycloheximide effect on carbon tetrachloride hepatotoxicity. Clin. Chim. Acta, 154, 181-189.

Pysz, M.A., Hao, F., Hizli, A.A., Lum, M.A., Swetzig, W.M., Black, A.R. and Black, J.D. (2014): Differential regulation of cyclin D1 expression by protein kinase $\mathrm{C} \alpha$ and $\epsilon$ signaling in intestinal epithelial cells. J. Biol. Chem., 289, 22268-22283.

Ramaiah, S.K. (2007): A toxicologist guide to the diagnostic interpretation of hepatic biochemical parameters. Food Chem. Toxicol., 45, 1551-1557.

Rosen, F., Roberts, N.R. and Nichol, C.A. (1959): Glucocorticosteroids and transaminase activity. I. Increased activity of glutamicpyruvic transaminase in four conditions associated with gluconeogenesis. J. Biol. Chem., 234, 476-480.

Tomimoto, D., Okuma, C., Ishii, Y., Akiyama, Y., Ohta, T., Kakutani, M., Ohkuma, Y. and Ogawa, N. (2015a): Pharmacological characterization of [trans-5'-(4-amino-7,7-
dimethyl-2-trifluoromethyl-7H-pyrimido[4,5-b][1,4]oxazin6-yl)-2', 3'-dihydrospiro(cyclohexane-1, 1'-inden)-4-yl] acetic acid monobenzenesulfonate (JTT-553), a novel acyl CoA:diacylglycerol transferase (DGAT) 1 inhibitor. Biol. Pharm. Bull., 38, 263-269.

Tomimoto, D., Okuma, C., Ishii, Y., Kobayashi, A., Ohta, T., Kakutani, M., Imanaka, T. and Ogawa, N. (2015b): JTT-553, a novel Acyl CoA:diacylglycerol acyltransferase (DGAT) 1 inhibitor, improves glucose metabolism in diet-induced obesity and genetic T2DM mice. J. Pharmacol. Sci., 129, 51-58.

Ulluwishewa, D., Anderson, R.C., McNabb, W.C., Moughan, P.J., Wells, J.M. and Roy, N.C. (2011): Regulation of tight junction permeability by intestinal bacteria and dietary components. J. Nutr., 141, 769-776.

United States Department of Agriculture (USDA). (2017-2018): Food and nutrient database for dietary studies, Corn oil. Available: https://fdc.nal.usda.gov/fdc-app.html\#/fooddetails/1103858/nutrients

Yang, C. and Kazanietz, M.G. (2003): Divergence and complexities in DAG signaling: looking beyond PKC. Trends Pharmacol. Sci., 24, 602-608.

Yen, C.L. and Farese, R.V. Jr. (2003): MGAT2, a monoacylglycerol acyltransferase expressed in the small intestine. J. Biol. Chem., 278, 18532-18537.

Yokoyama, H., Kobayashi, A., Kondo, K., Oshida, S.I., Takahashi, T., Masuyama, T., Shoda, T. and Sugai, S. (2018): A pharmacologic increase in activity of plasma transaminase derived from small intestine in animals receiving an acyl CoA: diacylglycerol transferase (DGAT) 1 inhibitor. J. Toxicol. Sci., 43, 135-157. 